\title{
COMMENTARY
}

\section{Standardizing data collection in severe trauma: call for linking up}

\author{
Doortje C Engel* \\ See related research by Ringdal et al., http://ccforum.com/content/15/5/R237
}

\begin{abstract}
Standardization of data collection in severely injured trauma patients in order to find the best performance and practice has been an issue for more than 20 years. The incidence of trauma has decreased and outcomes have improved over the past decades. Trauma still remains an important public health problem, however, and is listed by the World Health Organization as a leading cause of death and disability. Ringdal and colleagues prove the feasibility on a basic level in their prospective, intercontinental study showing the results of the Utstein Trauma Template. In-depth analysis is currently only partially possible. The future of standardizing data collection in trauma looks bright. However, bridging and cross-linking is necessary to a great extent in the future.
\end{abstract}

Trauma, despite efficacy of prevention measures, is still ranked in the top six causes of mortality and disability. As the world has become an open-source medical research space, it invites clinical researchers to try and compare their healthcare methods and performances between countries. Even the European Union advises strongly to benchmark national healthcare in several greater themes according to the European Community Health Indicators $[1,2]$. In trauma care, best practice for both pre-hospital care and in-hospital care of severely injured trauma patients has been subject to ongoing discussion for decades. Large differences in documentation between countries and registries pose challenges when comparing healthcare systems.

Ringdal and colleagues describe the international validation of the Utstein Trauma Template [3]. This template was formed through consensus of an international

*Correspondence: DoortjeEngel@gmail.com

Department of Neurosurgery, Cantonal Hospital of St Gallen, Rorschacherstrasse 95, CH-9007 St Gallen, Switzerland working group on systematic documentation of trauma care and performance in 2008 [4] using derivatives of the Utstein style recommendations [5] and the Scandinavian (SCANTEM), British (TARN), German (DGU-TR) and Italian (RITG) trauma registries. The overall aim of the commented article was to investigate the feasibility of this template to allow valid epidemiological trauma studies, to make it possible to identify important factors that are related to outcome in existing systems, and to identify systems or part of systems with high or low performance.

Complete data for 28 of 36 variables in $>80 \%$ of 962 patients from 42 centers in three continents (Europe, Australia and North America) are probably the best score ever in a prospective study of this dimension. The need for trauma registries was noticed in 1989 [6]. Ten years later the effectiveness of systematic reviews of trauma registries in order to improve performance in trauma care was proven [7]. Variables such as age, gender and the Abbreviated Injury Score seem to form no problems and are documented in $100 \%$ of cases, although the version of the Abbreviated Injury Score used does differ between centers. Labor-intensive parameters such as arterial base excess and the pre-hospital respiratory rate are still worrisome, with less than $50 \%$ completeness.

Despite the positive aspects of Ringdal and colleagues' study, a particular concern is the substantial variability in timing of outcome assessment, including recording of mortality. The Utstein Trauma Template mandated the use of 30-day survival as a short-term outcome variable. Eleven of the 24 centers collecting this variable (46\%) did not adhere to this definition, and instead recorded either outcome at hospital discharge, the in-hospital 30-day outcome or the outcome at the end of the total somatic stay. This variability of assessment of outcome is of particular concern when the intent of a standardized data collection is to facilitate comparison between centers and to benchmark the quality of healthcare delivery. A fixed point in time for outcome assessment becomes essential. If in-hospital mortality is taken as the endpoint (by definition at a variable point in time), a biased estimate leading to a false low mortality rate may result when, for 
example, patients with a poor prognosis are transferred early to other care facilities. Interestingly, the Utstein template also requires assessments of the Glasgow Outcome Scale as an outcome measure. The Glasgow Outcome Scale was introduced as a global functional scale for assessing outcome in patients after traumatic brain injury. Whether the Glasgow Outcome Scale is appropriate for use in general trauma is debatable, and furthermore the validity of the Glasgow Outcome Scale for assessing outcome for in-hospital patients can be challenged. By definition these patients are still dependent on care and thus cannot be judged as being independent. The performance of the Glasgow Outcome Scale as an outcome measure in general trauma requires further study.

Benchmarking quality of care is a highly relevant issue and standardization of data collection is essential. Another initiative on standardization of data collection is the international interagency effort on common data elements for traumatic brain injury initiated by the US National Institutes of Health $[8,9]$. The goals of this process have been discussed in a commentary in this journal [10] and have subsequently been published $[11,12]$ and are currently being broadly implemented. The initiatives of the Utstein Trauma Template further demonstrate broad support and feasibility for establishing consensus on recording of variables in trauma registries [13]. These efforts should preferably be compatible with other efforts towards standardization such as the Common Data Elements initiative in traumatic brain injury $[8,9]$. International, multidisciplinary collaboration and a lot of patience will be needed to reach consensus and to coordinate different visions and opinions when establishing recommendations for a core set of trauma data to be collected in both registries and studies.

Competing interests

The author declares that he has no competing interests.
Published: 12 January 2012

\section{References}

1. European Community Health Indicators Monitoring Joint Action 20092011 [http://ec.europa.eu/health/indicators/echi/index_en.htm]

2. Harbers M, Van der Wilk EA, Kramers PGN, Kuunders MMAP, Verschuuren M, Eliyahu H, Achterberg PW: Dare to Compare - Benchmarking Dutch Health with the European Community Health Indicators (ECHI). Bilthoven: RIVM; 2009.

3. Ringdal KG, Lossius HM, Jones JM, Lauritsen JM, Coats TJ, Palmer CS, Lefering R, Di Bartolomeo S, Dries DJ, Søreide K; The Utstein Trauma Data Collaborators: Collecting core data in severely injured patients using a consensus trauma template: an international multicentre study. Crit Care 2011, 15:R237.

4. Ringdal KG, Coats TJ, Lefering R, Di Bartolomeo S, Steen PA, Røise O, Handolin L, Lossius HM; Utstein TCD Expert Panel: The Utstein template for uniform reporting of data following major trauma: a joint revision by SCANTEM, TARN, DGU-TR and RITG. Scand J Trauma Resusc Emerg Med 2008, 16:7

5. Dick WF, Basket PJ: Recommendations for uniform reporting of data following major trauma - the Utstein style. A report of a working party of the International Trauma Anaesthesia and Critical Care Society (ITACCS). Resuscitation 1999, 42:81-100.

6. Pollock DA, McClain PW: Trauma registries. Current status and future prospects. JAMA 1989, 262:2280-2283.

7. Jurkovich GJ, Mock C: Systematic review of trauma system effectiveness based on registry comparisons. J Trauma 1999, 47:S46-S55.

8. NINDS Common Data Elements [www.commondataelements.ninds.nih. gov]

9. International Mission for Prognosis and Analysis of Clinical Trials in TBI [www.tbi-impact.org]

10. Maas Al: Standardisation of data collection in traumatic brain injury: key to the future? Crit Care 2009, 13:1016.

11. Maas Al, Harrison-Felix CL, Menon D, Adelson PD, Balkin T, Bullock R, Engel DC, Gordon W, Langlois-Orman J, Lew HL, Robertson C, Temkin N, Valadka A, Verfaellie M, Wainwright M, Wright DW, Schwab K: Standardizing data collection in traumatic brain injury. J Neurotrauma 2011, 28:177-187.

12. Thurmond VA, Hicks R, Gleason T, Miller AC, Szuflita N, Orman J, Schwab K: Advancing integrated research in psychological health and traumatic brain injury: common data elements. Arch Phys Med Rehabil 2010, 91:1633-1636.

13. European Trauma Registry Network [www.eurotrauma.net]

doi:10.1186/cc10561

Cite this article as: Engel DC: Standardizing data collection in severe

trauma: call for linking up. Critical Care 2012, 16:105 